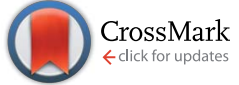

Cite this: RSC Adv., 2015, 5, 50638

Received 9th April 2015

Accepted 22nd May 2015

DOI: $10.1039 / c 5 r a 06306 j$

www.rsc.org/advances

\section{Flexible and in situ fabrication of nanochannels with high aspect ratios and nanopillar arrays in fused silica substrates utilizing focused ion beam $\dagger$}

\author{
Yan $\mathrm{Xu}^{*}$ and Nobuhiro Matsumoto
}

\begin{abstract}
Nanochannels fabricated in fused silica substrates are ideal tools for single (bio)molecular studies in biology and are promising in the development of innovative applications in chemistry. To obtain a higher throughput and a higher level of integration and functionalization, nanochannels with high aspect ratios and nano-in-nano structures are very much desired, but their fabrication is a challenge. Herein, we report a method for fabricating nanochannels with such challenging critical dimensions and structures in fused silica substrates by using a focused ion beam.
\end{abstract}

Nanofluidic devices, which are microchips incorporating nanometer-scale channels (i.e., nanochannels), are ideal tools to understand behaviors and phenomena of liquids and (bio) molecules confined in stringent nanospaces. ${ }^{\mathbf{1 - 4}}$ For example, confining a liquid in a nanochannel shows promise in approaching an ultimate goal to precisely handle and characterize single molecules. ${ }^{5-7}$ Instead of measuring averaged behavior of ensemble molecules, such single molecular approaches are promising for gaining an insight into fundamental phenomena. In addition, recent studies revealed altered properties of water confined in a nanochannel, with higher viscosity, ${ }^{\mathbf{8} 9}$ higher proton mobility, ${ }^{\mathbf{1 0}, 11}$ and lower dielectric constant $^{8}$ than in the bulk. Such unique properties are very promising to eventually bring epoch-making applications. These applications may include, for example, the realization of ultra-fast chemical reactors, ultra-high-efficiency energy converters, and ultra-high-sensitive analytical devices. Nevertheless, despite the increased exciting progress, the field of nanofluidics is still nascent, in contrast to its thriving 'brother' microfluidics. One critical reason is that the capability of

Nanoscience and Nanotechnology Research Center, Research Organization for the 21st Century, Osaka Prefecture University, 1-2, Gakuen-cho, Naka-ku, Sakai, Osaka 599-8570, Japan. E-mail: y-xu@21c.osakafu-u.ac.jp; Fax: +81-72-254-7813; Tel: +8172-254-7813

† Electronic supplementary information (ESI) available: Experimental section. See DOI: $10.1039 / \mathrm{c} 5 \mathrm{ra06306j}$ excellent control of the geometry of nanochannels is still lacking.

Nanochannels are the 'hearts' of nanofluidic devices and their fabrication varies depending on the materials. In the early days of development, nanochannels were fabricated on semiconductor wafers such as silicon, by directly transferring the well-established nanofabrication experience accumulated in microelectronics over several decades. ${ }^{12-14}$ In the past few years, the fabrication of nanochannels in fused silica substrates was achieved, using several tools as described later. After that, insulating fused silica immediately replaced semiconducting silicon as a major substrate material for fabrication of nanofluidic devices, in addition to a few polymeric substrates ${ }^{15-17}$ to a lesser extent. Fused silica is a glass consisting of silica in amorphous form. Fused silica possesses excellent properties such as superior optical transparency, chemical/biological inertness, thermal stability, and mechanical robustness. Furthermore, owing to their hydrophilic nature and well-established surface chemistry through a variety of silanization processes, fused silica nanochannels make it easy to perform liquid introduction and inner wall surface modification. These excellent properties are ideal and indispensable for chemical and biological studies, as well as applications at the nanoscale or single-molecular level under nanofluidic conditions.

Several tools have enabled the fabrication of nanochannels in fused silica substrates, with low aspect ratios (depths/widths) or unity (i.e., close to 1) aspect ratios. Standard photolithography incorporated with short-time wet or dry etching allows fabricating fused silica nanochannels with micrometer-scale widths and nanometer-scale depths, defined by the practical resolution limits of photolithography and duration of etching, respectively. ${ }^{18-20}$ Such nanochannels with low aspect ratios are also called planar nanochannels and have been used in early studies of DNA extension. Electron beam lithography (EBL) coupled with dry etching allows fabricating fused silica nanochannels with unity aspect ratios, namely, square nanochannels. ${ }^{21-24}$ The dimensions (i.e., widths and depths) of the 
square nanochannels fabricated by this method usually range from tens to hundreds of nanometers. This range is critically important for studies in nanofluidics because continuum assumptions for the Navier-Stokes (NS) equations, ${ }^{25}$ which are governing equations in fluid mechanics, still apply. The use of square nanochannels with dimensions in this important range has accelerated studies in nanofluidics, giving birth to the aforementioned discoveries of altered properties of water confined in nanospaces. More recently, an interesting study using sub-5 $\mathrm{nm}$ square nanochannels fabricated by focused ion beam (FIB) has extended the possibility of nanochannels to ultimate dimensions below $5 \mathrm{~nm},{ }^{26}$ where the NS equations no longer apply and liquids can be no longer defined as fluids.

In comparison with low-aspect-ratio planar nanochannels and near-unity-aspect-ratio square nanochannels, high-aspectratio nanochannels offer advantages including a higher throughput and a higher level of integration, but their fabrication is even more challenging. Only a few groups have reported the fabrication of high-aspect-ratio nanochannels in silicon ${ }^{27,28}$ and polymeric substrates ${ }^{15}$ using interferometric lithography, proton beam lithography, and others. Nevertheless, the fabrication of high-aspect-ratio nanochannels in fused silica substrates is still a remaining challenge.

In addition, the capability of further defining nanometerscale geometries inside nanochannels, namely, nano-in-nano structures, promises to bring about more powerful nanochannels with versatile integrated functions. Nanochannels with flat geometries have been widely used to date in a variety of fundamental phenomenon studies. In order to obtain deeper insights into those fundamental phenomena at a more precise level and fully utilize those new findings to develop the aforementioned epoch-making applications, nano-in-nano structures are desired. However, the fabrication of nano-in-nano structured nanochannels in fused silica substrates by using EBL coupled with dry etching needs a super-high-precision alignment technique during multiple-step nanofabrication, ${ }^{29}$ which unfortunately is a great hurdle.

Herein, we report a method for the fabrication of nanochannels with high aspect ratios and nanochannels with nanopillar arrays in fused silica substrates by utilizing FIB (Fig. 1). FIB has been much employed in the field of semiconductors for milling, deposition, and imaging of semiconducting materials but has seldom been applied to insulating materials. ${ }^{26,30}$ Our method enables the flexible and in situ fabrication of challenging nanochannel structures in insulating fused silica substrates through a conducting layer $(100 \mathrm{~nm}$ thick gold layer) pre-deposited on the substrate, under optimized conditions of beam parameters and processing parameters of FIB milling. The gold layer provides capabilities of (1) efficiently allowing sufficient conductance of charge during deep milling of the insulating fused silica substrate, (2) precisely defining the narrow channel feature to get a high aspect ratio, and (3) adequately protecting the surface of the substrate and/or the nanochannel in the case of nano-in-nano fabrication from material redeposition during FIB milling. In addition, the milling guided by simultaneous high-resolution FIB imaging allows positioning of target regions precisely. The high- precision positioning thereby enables the flexible and in situ fabrication of nano-in-nano structures such as nanopillar array nanochannels, without the need to additionally develop a highprecision alignment technique, which is very challenging. We believe that the fabrication method will contribute to the development of nanofluidic devices with a higher throughput and a higher level of integration and functionalization.

It is problematic to directly mill (or sputter) a fused silica substrate by using FIB, due to the charging effect caused by ion irradiation on the insulating substrate. In general, the charging effect arises from accumulation of static electric charges on the surface of a sample. In this study, gallium ion $\left(\mathrm{Ga}^{+}\right)$beams (Table 1) were used as ion beam sources. The irradiation of the insulating fused silica with $\mathrm{Ga}^{+}$ions will bring about the accumulation of excess positive charge on the surface. The charging effect adversely affects the quality of FIB milling, resulting in irregular milled features. We used a gold layer pre-deposited on the fused silica substrate as a conducting layer to overcome these charging problems. As one of the most sensitive materials for FIB milling processes, gold has a significantly high ion milling (or sputtering) yield (15.7 atoms/ion for $40 \mathrm{keV} \mathrm{Ga}^{+}$ions at normal incidence). ${ }^{31}$ The high milling yield offers a fast etching rate for the gold layer during FIB milling. In addition, focused $\mathrm{Ga}^{+}$ion beams are very suitable for milling gold and capable of milling features with vertical slope profiles in the gold layers. ${ }^{32}$ The fast etching rate together with the vertical etching profile thereby enabled quick transfer of a designed pattern feature (i.e., acting as a mask) to the fused silica layer, with high accuracy. This quickly formed gold mask then played a critical role in maintaining efficient conductance of charge for the $\mathrm{Ga}^{+}$ion beam to sufficiently mill the fused silica surface. In addition, the conducting gold layer could be easily removed by using a routine chemical etchant. After gold removal, a standard nanofluidic device could be obtained by bonding the

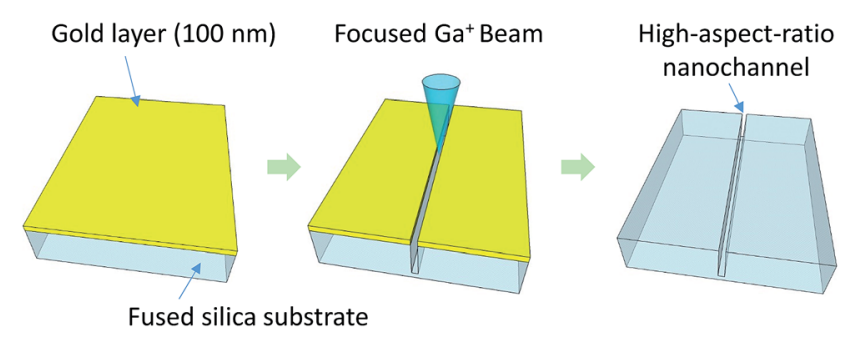

Fig. 1 Schematic diagram of the FIB milling process for the fabrication of high-aspect-ratio nanochannels.

Table 1 Aperture sizes, beam diameters, beam currents, and current densities of $40 \mathrm{keV} \mathrm{Ga}{ }^{+}$beams used in this study

\begin{tabular}{lllll}
\hline Beam no. & $\begin{array}{l}\text { Aperture } \\
\text { size }(\mu \mathrm{m})\end{array}$ & $\begin{array}{l}\text { Beam } \\
\text { diameter }(\mathrm{nm})\end{array}$ & $\begin{array}{l}\text { Beam } \\
\text { current }(\mathrm{nA})\end{array}$ & $\begin{array}{l}\text { Current density } \\
\left(\mathrm{A} \mathrm{cm}^{-2}\right)\end{array}$ \\
\hline 1 & 80 & 100 & 0.60 & 7.64 \\
2 & 40 & 48 & 0.13 & 7.18 \\
3 & 20 & 24 & 0.02 & 4.42
\end{tabular}


substrate containing the FIB-milled nanochannel structures and a substrate containing photolithographically fabricated microchannels through conventional fused silica bonding methods. ${ }^{3,33}$

Gold layers with different thickness were tested. A gold layer $100 \mathrm{~nm}$ thick was utilized in this study, decided by the milling capability and reproducibility. To verify the capability of milling, the fabrication of nanochannels with large critical dimensions (500 $\mathrm{nm}$ in width) was performed under routine FIB milling conditions (40 keV accelerating voltage, $40 \mu \mathrm{m}$ aperture, $48 \mathrm{~nm}$ beam diameter, $0.13 \mathrm{nA}$ beam current). Here, $40 \mathrm{keV}$ is a standard accelerating voltage generally used in the FIB milling mode. The beams were scanned over designed rectangular pattern regions with widths of $500 \mathrm{~nm}$, which were equal to the desired nanochannel widths. Owing to the assistance of the conducting gold layer, nanochannels approximately $500 \mathrm{~nm}$ wide were obtained as desired, with varying depths dependent on dwell times (Fig. 2). Here, the dwell time is a critical processing parameter capable of controlling FIB milling. During FIB milling, the FIB spot with a certain diameter (i.e., beam diameter) raster scans the sample surface within the region of a designed graphical pattern. The pattern is composed of graphical pixels that specify the location of the beam spot. The dwell time (typically $\mu$ s to $\mathrm{ms}$ ) defines the beam spot irradiation time at each pixel. As shown in Fig. 2, the depths of the milled nanochannels increase with an increase of dwell time, indicating that a longer dwell time could improve milling yields in the vertical direction in the fused silica layer.

In addition to dwell time, ion beam parameters such as aperture size, beam diameter, beam current, and current density were further verified and optimized for the fabrication of high-aspect-ratio nanochannels in fused silica substrates. The beam current (typically pA to $\mathrm{nA}$ ) is directly determined by aperture size $(\mu \mathrm{m})$ and condenser lens in the optical system of FIB. The current density $\left(\mathrm{A} \mathrm{cm}^{-2}\right)$ correlates with the beam diameter (nm) and the beam current. The values of the

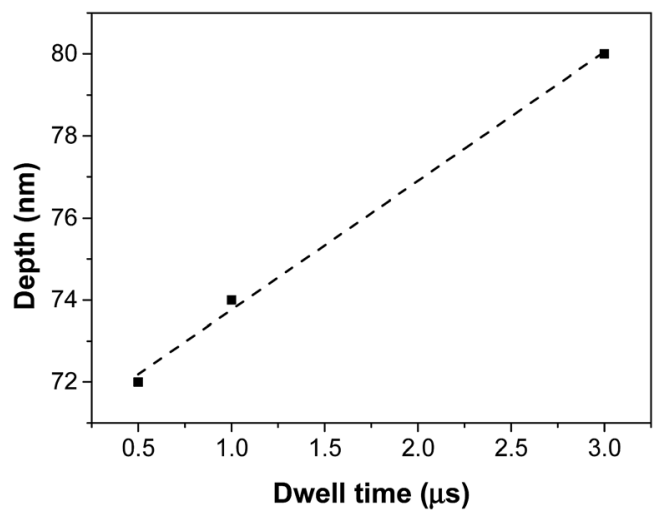

Fig. 2 The depth of the FIB-milled nanochannel as a function of the dwell time for the fabrication of nanochannels with large critical dimensions (design width $=500 \mathrm{~nm}$ and setting depth $=100 \mathrm{~nm}$ ) in fused silica substrates under routine FIB milling conditions $(40 \mathrm{keV}$ focused $\mathrm{Ga}^{+}$beam with a beam diameter of $48 \mathrm{~nm}$ and a beam current of $0.13 \mathrm{nA}$ ). corresponding beam parameters of $40 \mathrm{keV} \mathrm{Ga}^{+}$beams utilized in this study are listed in Table 1. In this experiment, we designed the widths of rectangular patterns to be $100 \mathrm{~nm}$ and set the depths for milling to be $600 \mathrm{~nm}$ (i.e., $500 \mathrm{~nm}$ for the fused silica layer), to obtain $100 \mathrm{~nm}$ wide and $500 \mathrm{~nm}$ deep fused silica nanochannels after removing the $100 \mathrm{~nm}$ gold layer. The setting depth is a processing parameter which can control the amount of milling. For a given setting depth, the number of beam scans over a pattern is directly dependent on the dwell time and the fabrication constant $\left(\mu \mathrm{m}^{3} \mathrm{nA}^{-1} \mathrm{~s}^{-1}\right)$, which describes the FIB milling efficiency in a certain material. In the case where the fabrication constant of materials is well established, for example silicon, theoretically the actual depth of milling should be approximately equal to the setting depth. However, for the gold/fused silica two-layer system, the fabrication constant is difficult to determine. To make things simpler, in this study we assumed the fabrication constant to be $0.28 \mu \mathrm{m}^{3} \mathrm{nA}^{-1} \mathrm{~s}^{-1}$, which is equal to that of silicon for $40 \mathrm{keV}$ $\mathrm{Ga}^{+}$ion beam milling. It should be noted that the actual fabrication constant of the gold/fused silica system may not be $0.28 \mu \mathrm{m}^{3} \mathrm{nA}^{-1} \mathrm{~s}^{-1}$. By using the assumed fabrication constant, the actual depth becomes only dependent on the dwell time in a given beam condition.

Fig. 3 shows the result of the optimization of the beam diameter and the dwell time for milling of desired high-aspectratio nanochannels $(100 \mathrm{~nm}$ in width, $500 \mathrm{~nm}$ in depth, aspect ratio $=5.0$ ). In Fig. 3, width (actual)/width (design) represents the ratio between the actual width and the design width $(100 \mathrm{~nm})$, depth (actual)/depth (setting) represents the ratio between the actual depth and the setting depth $(500 \mathrm{~nm})$, in the fused silica layer. In the case where both these ratios are near 1.0, the nanochannels as prescribed are considered to be obtained. The widths of the milled nanochannels were dependent on both the beam diameter and the dwell time. The larger the beam diameter, the wider was the milled nanochannel (Fig. 3a). Among the three beam conditions utilized in this study (Table 1), the ion beam with the smallest beam diameter ( $24 \mathrm{~nm}$; beam 3 in Table 1) enabled milling of the nanochannels with actual widths closest to $100 \mathrm{~nm}$, at each dwell time $(0.5 \mathrm{~s}, 10 \mathrm{~s}$, and $100 \mathrm{~s})$. For the ion beam with a diameter of $24 \mathrm{~nm}$, increasing the dwell time resulted in an increase of the actual depth (Fig. 3b) and thereby an increase of the aspect ratio (Fig. 3c). Nanochannels with high aspect ratios ranging from $c a$. 3.0 to 5.0 were obtained by using ion beams with a diameter of $24 \mathrm{~nm}$ (Fig. 3c). Moreover, at the dwell time of $100 \mu \mathrm{s}$ in this case, both the ratio of width (actual)/width (design) and the ratio of depth (actual)/depth (setting) were approximately equal to 1 (Fig. 3a and b). These results revealed that the conditions of $40 \mathrm{keV} \mathrm{Ga}{ }^{+}$ion beam, $24 \mathrm{~nm}$ beam diameter, $100 \mu$ s dwell time were optimal FIB milling conditions to obtain nanochannels as prescribed for the gold/fused silica system, using the assumed fabrication constant $\left(0.28 \mu \mathrm{m}^{3} \mathrm{nA}^{-1} \mathrm{~s}^{-1}\right)$.

Fig. 4 shows atomic force microscopy (AFM) images and scanning ion microscope (SIM) images for a nanochannel $c a$. $500 \mathrm{~nm}$ deep and $c a .100 \mathrm{~nm}$ wide (Fig. 4a and b) and a nanochannel ca. $500 \mathrm{~nm}$ deep and ca. $500 \mathrm{~nm}$ wide (Fig. 4c and d) which were fabricated using the optimal conditions. While both 
(a)

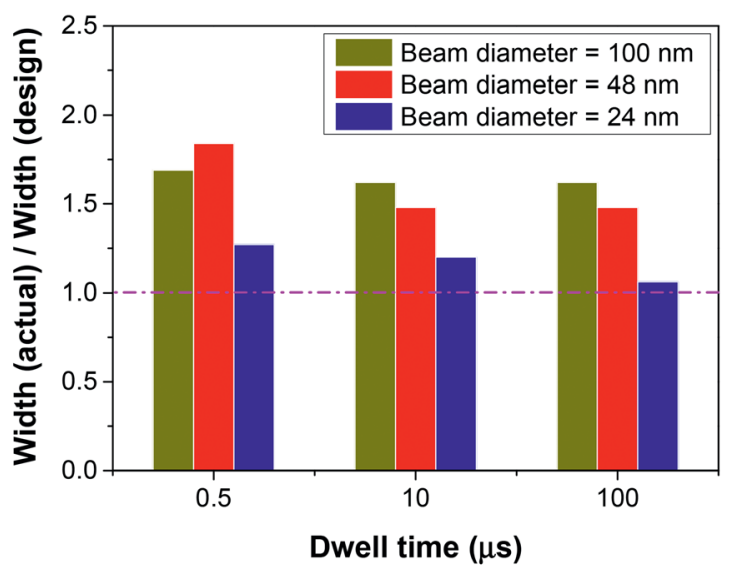

(b)

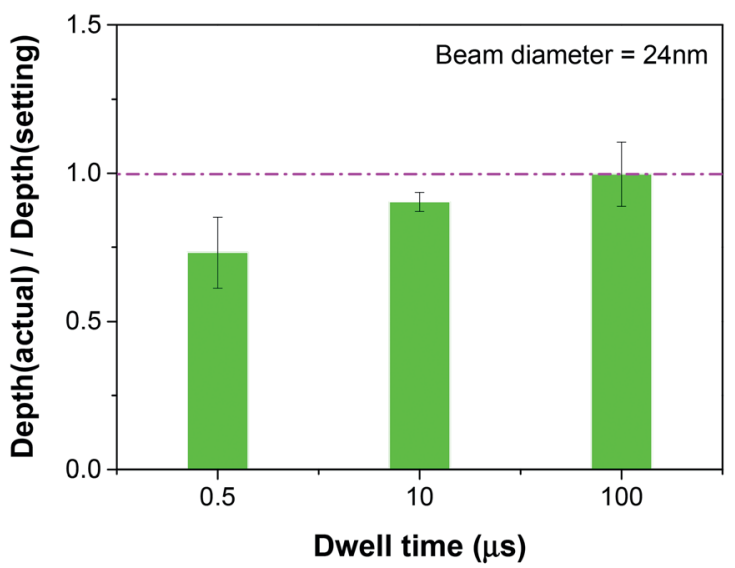

(c)

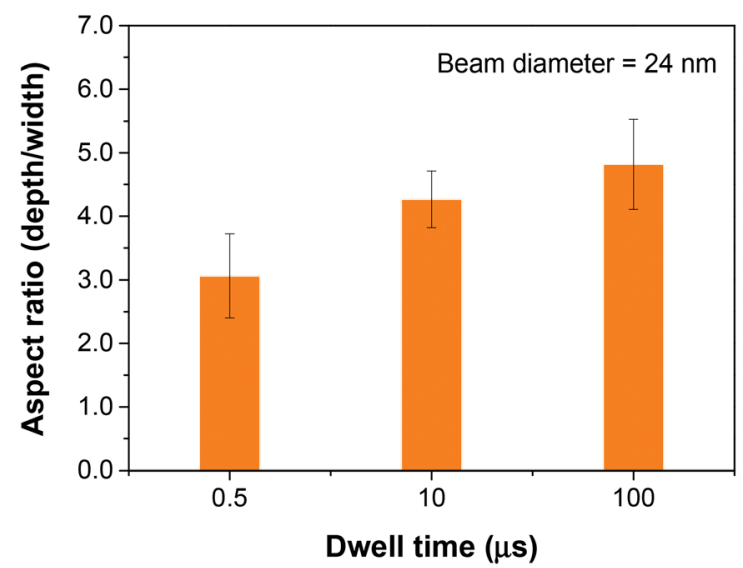

Fig. 3 Optimization of the beam diameter and the dwell time for milling high-aspect-ratio nanochannels (design width $=100 \mathrm{~nm}$ and setting depth $=500 \mathrm{~nm}$ ) in fused silica substrates. (a) Width (actual)/ width (design) of the nanochannels fabricated using $40 \mathrm{keV} \mathrm{Ga}^{+}$ion beams with different beam diameters at varying dwell times. (b) Depth (actual)/depth (setting) and (c) aspect ratios (depths/widths) of the nanochannels fabricated using the ion beam with a beam diameter of $24 \mathrm{~nm}$ at varying dwell times. Data are mean $\pm S D, n \geq 3$.

the width and the depth of the wide nanochannel (500 $\mathrm{nm}$ deep and $500 \mathrm{~nm}$ wide) could be roughly characterized from its AFM image (Fig. 4c), the depth of the narrow nanochannel (500 nm deep and $100 \mathrm{~nm}$ wide) was difficult to determine only from its AFM image (Fig. 4a), due to the larger size (several tens of micrometers) of the cantilever of the AFM than that of the nanochannel. In addition, it was also difficult to obtain the cross-sectional shapes of the nanochannels using the AFM for the same reason. The SIM was used to characterize the crosssectional shapes of the nanochannels. The SIM images were obtained using an observation mode of our FIB system. An extremely small beam current, lower than $0.01 \mathrm{nA}$, was used for the SIM observation to avoid damage to the surface of the specimens resulting from ion beam scanning. The crosssectional shapes of the nanochannels (Fig. $4 \mathrm{~b}$ and d) were obtained from the observation of the FIB-milled cross-sections tilted $60^{\circ}$ with respect to the ion beam. The SIM images revealed that the side walls of both nanochannels were near vertical $\left(5-7^{\circ}\right)$. While the $500 \mathrm{~nm}$ deep and $100 \mathrm{~nm}$ wide nanochannel had a roughly square cross-section, the $500 \mathrm{~nm}$ deep and $500 \mathrm{~nm}$ wide nanochannel exhibited a quite sharp bottom due to narrowing of the channel.

Furthermore, the optimal beam conditions $\left(40 \mathrm{keV} \mathrm{Ga}{ }^{+}\right.$ion beam, $24 \mathrm{~nm}$ beam diameter, and $100 \mu$ s dwell time) were applied to fabricate nanochannels with nanopillar arrays (Fig. 5). The structures are nano-in-nano structures which are difficult to fabricate with current popular nanochannel fabrication tools for the reason mentioned above. In addition, while microchannels with nanopillars have been reported to possess improved bioseparation capabilities in comparison with microchannels without nanopillars, ${ }^{34,35}$ similar structures in nanochannels are greatly desired to further improve these capabilities at the nanoscale, but their fabrication is a critical issue. The fabrication of nanochannels with nanopillar arrays was achieved through either a flexible one-step FIB milling strategy (Fig. 5a) or an in situ two-step FIB milling strategy (Fig. 5b). For the former (Fig. 5a), the nanochannel and the nanopillars were simultaneously fabricated in a single FIB milling process, and therefore a nanochannel with nanopillars whose heights are approximately equal to the height (i.e., depth) of the nanochannel could be obtained, as shown in Fig. $5 \mathrm{c}$ and d. For the latter (Fig. 5b), the nanochannel and the nanopillars were sequentially fabricated through two independent millings in situ, and therefore a nanochannel with nanopillars whose heights are less than the height (i.e., depth) of the nanochannel could be obtained, as shown in Fig. 5e and f. Nanopillars with sizes (widths or diameters) ranging from hundreds (Fig. 5c-e) to tens (Fig. 5f) of nanometers as well as aspect ratios (heights/ sizes) varying from 0.9 (Fig. 5e) to 2.5 (Fig. 5c) were successfully fabricated in both planar (Fig. 5c-e) and square (Fig. 5f) nanochannels. The density of the nanopillar array, which is characterized by the ratio of the sizes of nanopillars to the space between nanopillars (width/space, in short), could be flexibly adjusted. For example, Fig. 5c shows a SIM image of a nanochannel with a low-density (size/space of $c a$. 0.2) nanopillar array, and Fig. 5d-f show SIM images of nanochannels with relatively high-density (width/space of $c a$. 1.0-1.1) nanopillar arrays. These results revealed that both milling strategies enabled the flexible and in situ fabrication of nanopillar array nanochannels with prescribed sizes, aspect ratios, and densities 

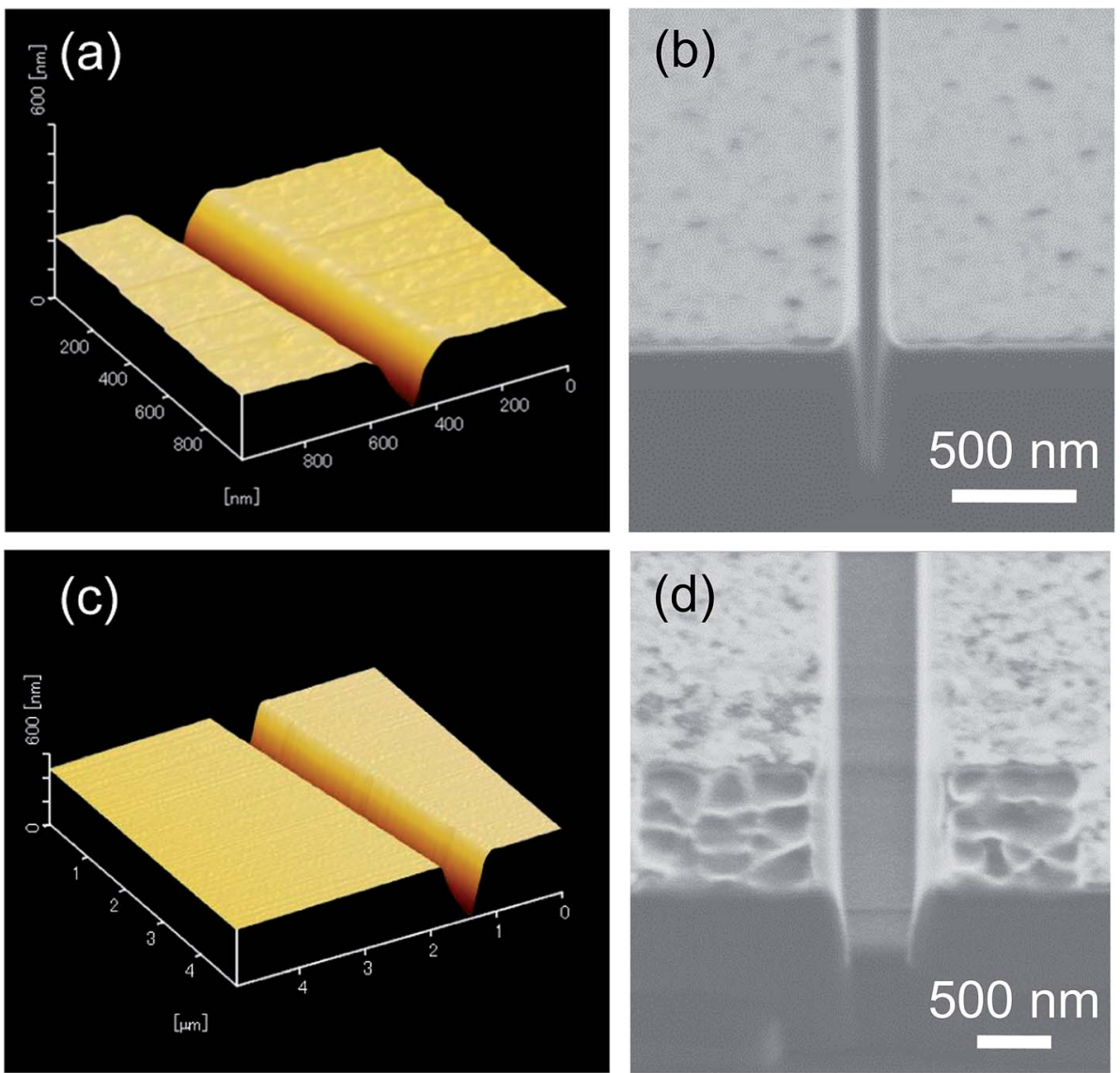

Fig. 4 AFM images and SIM images for ( $a$ and b) a nanochannel ca. $500 \mathrm{~nm}$ deep and ca. $100 \mathrm{~nm}$ wide and (c and d) a nanochannel ca. $500 \mathrm{~nm}$ deep and ca. $500 \mathrm{~nm}$ wide, which were fabricated using the optimal conditions. For (b and d) SIM images, FIB-milled cross-sections of nanochannels were tilted $60^{\circ}$ with respect to the ion beam. Note: the bumps on the surface near the cross-section shown in (d) resulted from the FIB milling for obtaining the cross-section of the nanochannel.

(a)
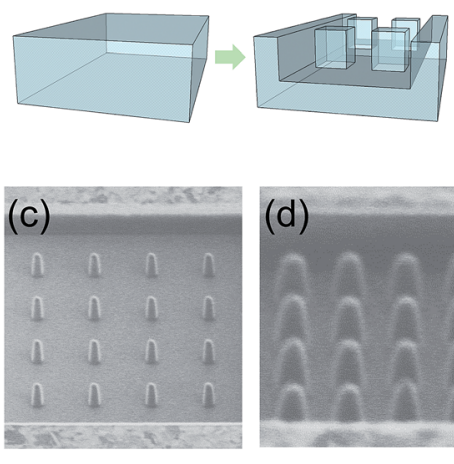

$\overline{1.0 \mu \mathrm{m}}$

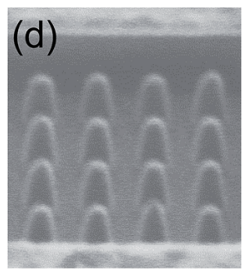

$500 \mathrm{~nm}$ (b) $\quad 1^{\text {st }}$ FIB milling

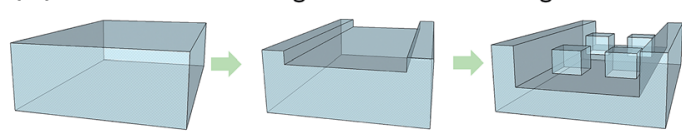

$2^{\text {nd }}$ FIB milling

(e)

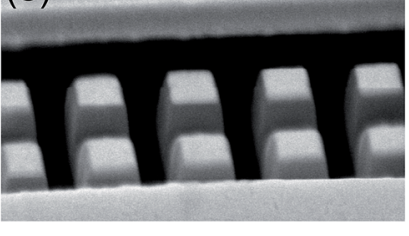

$1.0 \mu \mathrm{m}$

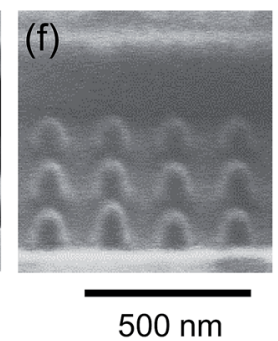

Fig. 5 FIB milling strategies ( $a$ and b) and SIM images (c-f) of nanochannels with nanopillar arrays. (a) The one-step milling strategy. (b) The twostep milling strategy. (c) A low-density nanopillar (180 nm wide, $450 \mathrm{~nm}$ high, aspect ratio $=2.5$, spaced by $900 \mathrm{~nm}$ ) array in a nanochannel with a depth of $450 \mathrm{~nm}$, and (d) a high-density nanopillar ( $150 \mathrm{~nm}$ wide, $320 \mathrm{~nm}$ high, aspect ratio = 2.1, spaced by $150 \mathrm{~nm}$ ) array in a nanochannel with a depth $320 \mathrm{~nm}$, which were fabricated using (a) the one-step FIB milling strategy. (e) A nanopillar (520 nm wide, $480 \mathrm{~nm}$ high, aspect ratio =0.9, spaced by $480 \mathrm{~nm}$ ) array in a planar nanochannel $(700 \mathrm{~nm}$ deep and $3 \mu \mathrm{m}$ wide), and (f) a nanopillar ( $80 \mathrm{~nm}$ wide, $120 \mathrm{~nm}$ high, aspect ratio $=1.5$, spaced by $120 \mathrm{~nm}$ ) array in a square nanochannel (400 nm deep, $800 \mathrm{~nm}$ wide), which were fabricated using (b) the two-step FIB milling strategy. 
of the nanopillars, as well as prescribed widths and depths of the nanochannels.

In conclusion, we presented a method for fabricating highaspect-ratio nanochannels as well as nanopillar array nanochannels in fused silica substrates. The method involved FIB milling of nanochannel structures through a $100 \mathrm{~nm}$ gold layer pre-deposited on the fused silica substrates, under optimized conditions of beam parameters and processing parameters. The use of a gold layer provided advantages of allowing flexibly and efficiently milling of deep and narrow nanochannels in the insulating fused silica substrates. With the method, the fabrication of nanochannels with high aspect ratios ranging from $c a$. 3.0 to 5.0 was achieved. Furthermore, in situ fabrication of nanochannels having nanopillar arrays with prescribed sizes ranging from hundreds to tens of nanometers, varying aspect ratios from $c a$. 0.9 to 2.5 , and desired densities in the nanochannels was accomplished. The method would be useful for developing integrated nanofluidic devices with high throughput.

\section{Acknowledgements}

This work was partially supported by JSPS KAKENHI grant numbers 26706010, 26630403, MEXT KAKENHI number 26107714, and the Asahi Glass Foundation.

\section{Notes and references}

1 W. Sparreboom, A. van den Berg and J. C. T. Eijkel, Nat. Nanotechnol., 2009, 4, 713-720.

2 A. Piruska, M. Gong, J. V. Sweedler and P. W. Bohn, Chem. Soc. Rev., 2010, 39, 1060-1072.

3 Y. Xu, K. Jang, T. Yamashita, Y. Tanaka, K. Mawatari and T. Kitamori, Anal. Bioanal. Chem., 2012, 402, 99-107.

4 T. Tsukahara, T. Maeda, A. Hibara, K. Mawatari and T. Kitamori, RSC Adv., 2012, 2, 3184-3186.

5 S. L. Levy and H. G. Craighead, Chem. Soc. Rev., 2010, 39, 1133-1152.

6 L. D. Menard, C. E. Mair, M. E. Woodson, J. P. Alarie and J. M. Ramsey, ACS Nano, 2012, 6, 9087-9094.

7 K. Frykholm, M. Alizadehheidari, J. Fritzsche, J. Wigenius, M. Modesti, F. Persson and F. Westerlund, Small, 2014, 10, 884-887.

8 A. Hibara, T. Saito, H. B. Kim, M. Tokeshi, T. Ooi, M. Nakao and T. Kitamori, Anal. Chem., 2002, 74, 6170-6176.

9 N. R. Tas, J. Haneveld, H. V. Jansen, M. Elwenspoek and A. van den Berg, Appl. Phys. Lett., 2004, 85, 3274-3276.

10 S. R. Liu, Q. S. Pu, L. Gao, C. Korzeniewski and C. Matzke, Nano Lett., 2005, 5, 1389-1393.

11 T. Tsukahara, A. Hibara, Y. Ikeda and T. Kitamori, Angew. Chem., Int. Ed., 2007, 46, 1180-1183.

12 J. Han and H. G. Craighead, J. Vac. Sci. Technol., A, 1999, 17, 2142-2147.

13 J. Haneveld, H. Jansen, E. Berenschot, N. Tas and M. Elwenspoek, J. Micromech. Microeng., 2003, 13, S62-S66.

14 J. L. Perry and S. G. Kandlikar, Microfluid. Nanofluid., 2006, 2, 185-193.
15 K. A. Mahabadi, I. Rodriguez, S. C. Haur, J. A. van Kan, A. A. Bettiol and F. Watt, J. Micromech. Microeng., 2006, 16, 1170-1180.

16 L. H. Thamdrup, A. Klukowska and A. Kristensen, Nanotechnology, 2008, 19, 125301.

17 R. Chantiwas, M. L. Hupert, S. R. Pullagurla, S. Balamurugan, J. Tamarit-Lopez, S. Park, P. Datta, J. Goettert, Y. K. Cho and S. A. Soper, Lab Chip, 2010, 10, 3255-3264.

18 A. Balducci, P. Mao, J. Y. Han and P. S. Doyle, Macromolecules, 2006, 39, 6273-6281.

19 J. D. Cross, E. A. Strychalski and H. G. Craighead, J. Appl. Phys., 2007, 102, 024701.

20 G. B. Salieb-Beugelaar, J. Teapal, J. van Nieuwkasteele, D. Wijnperle, J. O. Tegenfeldt, F. Lisdat, A. van den Berg and J. C. T. Eijkel, Nano Lett., 2008, 8, 1785-1790.

21 E. Tamaki, A. Hibara, H. B. Kim, M. Tokeshi, T. Ooi, M. Nakao and T. Kitamori, Anal. Sci., 2006, 22, 529-532.

22 W. Reisner, J. P. Beech, N. B. Larsen, H. Flyvbjerg, A. Kristensen and J. O. Tegenfeldt, Phys. Rev. Lett., 2007, 99, 058302.

23 M. Graczyk, M. Balaz, A. Kvennefors, H. Linke and I. Maximov, J. Vac. Sci. Technol., B: Nanotechnol. Microelectron.: Mater., Process., Meas., Phenom., 2012, 30, 06FF09.

24 K. Shirai, K. Mawatari and T. Kitamori, Small, 2014, 10, 1514-1522.

25 B. J. Kirby, Micro- and nanoscale fluid mechanics: transport in microfluidic devices, Cambridge University Press, New York, 2010.

26 L. D. Menard and J. M. Ramsey, Nano Lett., 2011, 11, 512517.

27 M. J. O'Brien, P. Bisong, L. K. Ista, E. M. Rabinovich, A. L. Garcia, S. S. Sibbett, G. P. Lopez and S. R. J. Brueck, J. Vac. Sci. Technol., B: Microelectron. Nanometer Struct.Process., Meas., Phenom., 2003, 21, 2941-2945.

28 A. L. Garcia, L. K. Ista, D. N. Petsev, M. J. O'Brien, P. Bisong, A. A. Mammoli, S. R. J. Brueck and G. P. Lopez, Lab Chip, 2005, 5, 1271-1276.

29 Y. Xu, N. Matsumoto, Q. Wu, Y. Shimatani and H. Kawata, Lab Chip, 2015, 15, 1989-1993.

30 W. X. Li, G. Lalev, S. Dimov, H. Zhao and D. T. Pham, Appl. Surf. Sci., 2007, 253, 3608-3614.

31 P. G. Blauner, J. S. Ro, Y. Butt and J. Melngailis, J. Vac. Sci. Technol., B: Microelectron. Process. Phenom., 1989, 7, 609-617. 32 K. P. Müller, Microelectron. Eng., 1990, 11, 443-447.

33 Y. Xu, C. X. Wang, Y. Y. Dong, L. X. Li, K. Jang, K. Mawatari, T. Suga and T. Kitamori, Anal. Bioanal. Chem., 2012, 402, 1011-1018.

34 T. Yasui, N. Kaji, M. R. Mohamadi, Y. Okamoto, M. Tokeshi, Y. Horiike and Y. Baba, ACS Nano, 2011, 5, 7775-7780.

35 T. Yasui, S. Rahong, K. Motoyama, T. Yanagida, Q. Wu, N. Kaji, M. Kanai, K. Doi, K. Nagashima, M. Tokeshi, M. Taniguchi, S. Kawano, T. Kawai and Y. Baba, ACS Nano, 2013, 7, 3029-3035. 